\title{
Mejora de la productividad en la elaboración de pisco puro no aromático
}

\author{
Lincoln Erwin Betalleluz Pallardel \\ Ingeniería Industrial n. 27, 2009, ISSN 1025-9929, pp. 51-60
}

\begin{abstract}
RESUMEN: La producción de pisco ha tenido en los últimos años un desarrollo notable y sostenido, desde su reconocimiento como denominación de origen peruano hasta el aprecio de su calidad en el mundo entero. Aun así, es posible mejorar el sistema de producción mediante la modificación de las condiciones de la fermentación, con ventajas de diverso tipo, como la mejor preservación de los sabores y el aroma de la uva, el mejoramiento del proceso de prensado, que permite obtener mayor cantidad de jugo y, la más importante, la posibilidad de incrementar la productividad de la uva en $50,98 \%$.
\end{abstract}

Palabras clave: uva / pisco / fermentación / productividad

\section{Improvement of the productivity in the production of pure non aromatic pisco}

ABSTRACT: The production of pisco has had a remarkable and sustained development during the last years, from its recognition as a denomination of Peruvian origin to the esteem of its quality throughout the world. Even so, it is possible to improve the production system by means of modification of the conditions of fermentation, with advantages of different kinds such as the best preservation of grape's flowering and aroma, the improvement of the pressing process which allows to obtain a higher quantity of juice and, the most importante, the possibility of increasing the productivity of the grape in $50,98 \%$.

Keywords: grape / pisco / fermentation / productivity 


\section{INTRODUCCIÓN}

Según la Norma Técnica Peruana NTP 211.0012006 el pisco "Es el aguardiente obtenido exclusivamente por destilación de mostos frescos de 'Uvas Pisqueras' recientemente fermentados, utilizando métodos que mantengan el principio tradicional de calidad establecido en las zonas de producción reconocidas". ${ }^{1}$

El Estado peruano ha reconocido el pisco como denominación de origen peruano, y como sus zonas de producción: Lima, Ica, Arequipa, Moquegua y los valles de Locumba, Sama y Caplina, en el departamento de Tacna.

Reforzando estos conceptos, Johnny Schuler expresa: "Pero el pisco es, creo yo, mucho más. Para mí es el mejor aguardiente de uva hecho en el mundo, porque en realidad es un destilado de puro jugo de uva, sin ningún otro ingrediente. Esto lo hace una bebida singular que se diferencia de otros licores por sus características particulares y únicas". ${ }^{2}$

Las estadísticas de la producción de pisco muestran un crecimiento muy marcado en los últimos años; según datos de la Comisión Nacional del Pisco (Conapisco) la evolución de la producción es como sigue:

\section{Cuadro 1}

Perú: Producción de pisco (estimada)

\begin{tabular}{ccc}
\hline Año & Volumen (millones de litros) & Fuente \\
\hline 2002 & 1,5 & Technoserve \\
2003 & 2,3 & SNI \\
2004 & 2,8 & Produce \\
2005 & 3,8 & Produce \\
2006 & 4,1 & Produce \\
2007 & 4,9 & Produce \\
2008 & $5,5-5,8$ & Conapisco \\
\hline
\end{tabular}

Fuente: <www.conapisco.org.pe>.

1 Indecopi. Norma Técnica Peruana NTP 211.001. Bebidas alcohólicas. Pisco. Requisitos. Lima, 2006.

2 Schuler, Johnny. Pasión por el pisco, 2004, p. 18. 
Como se aprecia, el futuro del pisco es muy prometedor. Frente a esta coyuntura, el autor se propone exponer algunas experiencias desarrolladas en la producción de pisco, así como introducir al lector en el maravilloso mundo de nuestra bebida de bandera.

\section{TIPOS DE PISCO}

De acuerdo con la variedad de la uva que se utilice en su fabricación, el pisco adquiere las siguientes denominaciones:

- Pisco puro no aromático.- Se obtiene exclusivamente de una sola variedad de uva pisquera, que no posee un aroma fuerte, como la uva quebranta.

- Pisco puro aromático.- Se produce a partir de una sola variedad de uva pisquera con aroma sobresaliente, por ejemplo la uva italia.

- Pisco mosto verde.- Es el pisco obtenido de la destilación de mostos frescos de uvas pisqueras con fermentación interrumpida.

- Pisco acholado. Es producto de una mezcla de uvas pisqueras, mostos o piscos.

\section{PROCESO DE PRODUCCIÓN DEL PISCO PURO NO AROMÁTICO CON UVA QUEBRANTA}

El proceso de producción del pisco puro no aromático se inicia cuando la uva quebranta llega a la planta de procesamiento, procedente del viñedo, para ser depositada en la tolva de la máquina estrujadora-despalilladora, en la que se estruja la uva y en forma simultánea se retiran los palillos o escobajos. El palillo o escobajo es el soporte del racimo que queda luego de retirar las uvas.

El producto del proceso anterior es transportado a una prensa donde, mediante un proceso de prensado mecánico se obtiene el jugo de la uva y se van separando las cáscaras, las pepas y algo de pulpa, a lo que se denomina orujo.

El jugo de uva así obtenido es luego conducido a unos tanques para el proceso de fermentación por alrededor de once días. Al inicio de la fermentación el jugo de uva tiene una densidad de $1096 \mathrm{~g} /$ litro aproximadamente, lo cual permite comprobar el contenido de azúcar. Después, por acción de las levaduras, que convierten el azúcar en alcohol, 
la densidad disminuye hasta un $995 \mathrm{~g} /$ litro. Durante la fermentación se controla de manera constante la densidad y la temperatura, mediante un mostímetro y un termómetro, para supervisar el proceso y determinar su término. Al jugo de uva fermentado se le denomina mosto.

Concluida la fermentación, se forman naturalmente unos sedimentos en la base de los tanques, los que son separados del mosto mediante el desencubado, que se realiza con una bomba.

Una vez concluida la etapa de fermentación, el mosto es trasladado al alambique, donde se lleva a cabo el proceso de destilación, que consiste en calentar el mosto para que por evaporación y condensación se obtenga el pisco. Al inicio del proceso de destilado se retira una parte determinada del alcohol condensado, denominado "cabeza", el cual tiene componentes no adecuados para la calidad del pisco.

Mientras continúa la destilación se controla constantemente el grado alcohólico, hasta que este alcance un valor de $42^{\circ} \mathrm{GL}$, lo cual determina el fin del proceso. El líquido que continúa saliendo, denominado "cola", es desechado. De esta manera, el pisco está constituido por la porción central o cuerpo del destilado.

A continuación, el pisco es depositado en tanques de acero inoxidable donde reposa durante tres meses, lapso en el que sufre transformaciones que mejoran su sabor y aroma.

Una vez concluida la fase de reposo del pisco, este es filtrado y envasado en botellas de $750 \mathrm{ml}$; se les colocan las tapas, las etiquetas $\mathrm{y}$, finalmente, son puestas en cajas con una capacidad de doce botellas cada una, las que posteriormente son trasladadas al almacén de productos terminados. Véase el diagrama 1, que muestra en forma esquemática el proceso descrito. 


\section{Diagrama 1}

Proceso para la elaboración de pisco puro no aromático

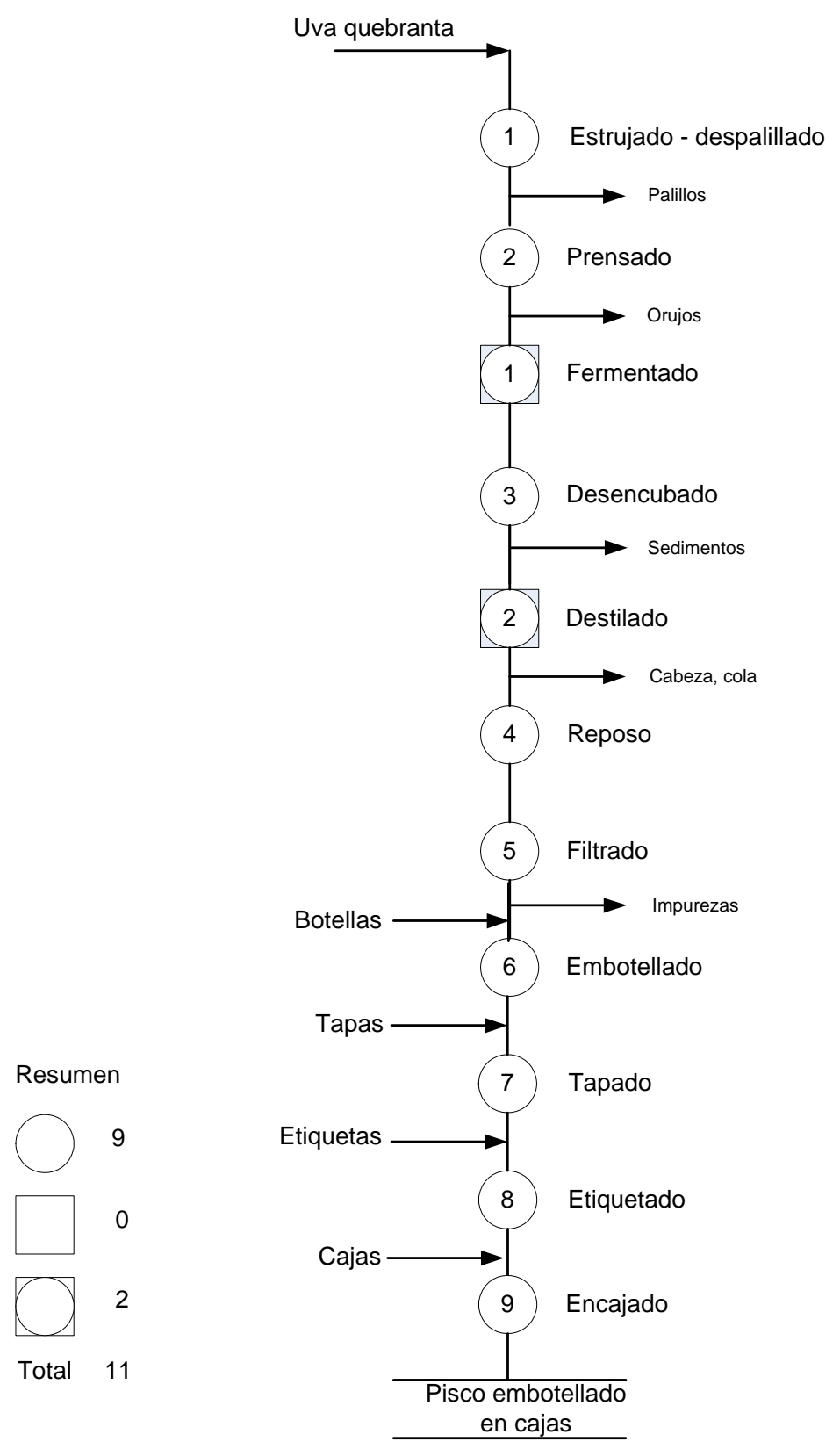




\section{MEJORA EN LA FERMENTACIÓN}

En lo que respecta a la fermentación del pisco, la Norma Técnica Peruana NTP 211.0012006 establece: "El proceso de fermentación puede realizarse sin maceración o con maceración parcial o completa de orujos de uvas pisqueras, controlando la temperatura y el proceso de degradación de los azúcares del mosto". ${ }^{3}$

Tomando en consideración lo establecido por la norma y la experiencia de diversos productores referida a la elaboración del pisco puro aromático, se probará una modificación del proceso, como se explica a continuación:

Luego de la fase de estrujado-despalillado, tanto el jugo como los orujos, constituidos por la pulpa, cáscaras y pepas, son transportados a un tanque donde permanecen por aproximadamente 36 horas, proceso que se denomina macerado. Concluido el tiempo indicado tiene lugar la labor de prensado para separar el jugo de uva, y continúa la fase de fermentación por once días.

Entre las ventajas que se obtienen por la modificación del proceso de elaboración del pisco se encuentran las siguientes:

- El pisco preserva de manera más completa los sabores y aromas de la uva.

- Favorece la fermentación de los azúcares de los orujos.

- El proceso de prensado se facilita y aumenta la cantidad de jugo que se obtiene.

- Se incrementa la productividad

3 Indecopi. Op. cit. 
Diagrama 2

Modificación del proceso para la elaboración de pisco puro no aromático

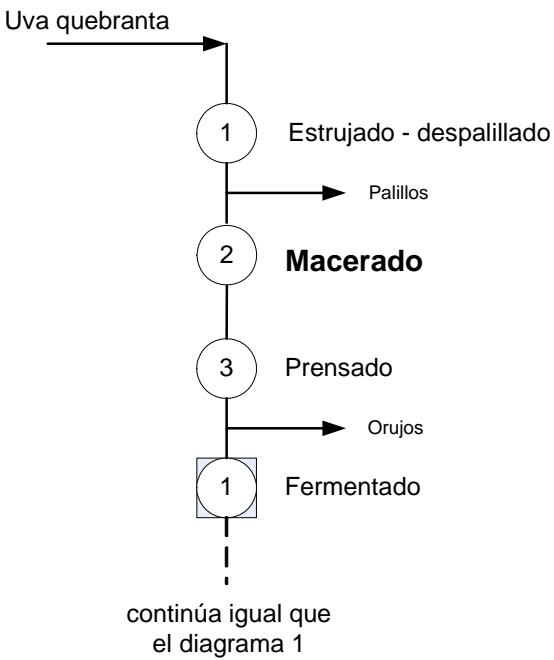

\section{RESULTADOS DE LA MEJORA}

En las siguientes páginas se describen las pruebas para la aplicación de la mejora, y se ofrecen cifras y datos de sus resultados.

Las producciones de prueba se realizaron en la bodega y el viñedo del autor, ubicados en Nuevo Imperial, provincia de Cañete, en el kilómetro 11,9 de la carretera a Lunahuaná. Se utilizaron los siguientes equipos:

- Estrujadora-despalilladora.

- Prensa mecánica diseñada por el autor en acero inoxidable, con un sistema de bombeo a los tanques de fermentación.

- Bomba para trasiego de jugo de uva y orujos.

- Tanques de fermentación de polietileno.

- Bomba para trasiego de mosto.

- Alambique de cobre calentado con gas, con un adecuado sistema de enfriamiento.

- Bomba para trasiego de pisco

- Tanques de reposo de acero inoxidable. 
Lincoln Betalleluz Pallardel

- Filtro, prensa y llenadora de botellas.

- Tapadora.

Prueba 1: Se realizó el proceso sin maceración, como se indica en el diagrama 1. Los resultados aparecen en el cuadro 2:

Cuadro 2

Resultado de las pruebas sin maceración

\begin{tabular}{lc}
\hline Concepto & Datos y resultado \\
\hline Fecha & Marzo-abril del 2006 \\
Cantidad de uva procesada & $7.050 \mathrm{~kg}$ \\
Volumen de pisco producido & 795 litros \\
\hline
\end{tabular}

Fuente: Elaboración propia.

Prueba 2: Se realizó la prueba aplicando el proceso con maceración, como se indica en el diagrama 2. Los resultados se muestran en el cuadro 3:

\section{Cuadro 3}

Resultado de las pruebas con maceración

\begin{tabular}{lc}
\hline Concepto & Datos y resultado \\
\hline Fecha & Marzo-abril del 2007 \\
Cantidad de uva procesada & $7.100 \mathrm{~kg}$ \\
Volumen de pisco producido & 1.209 litros \\
\hline
\end{tabular}

Fuente: Elaboración propia.

Con el fin de verificar que el pisco obtenido con la propuesta de mejora cumple con los requisitos establecidos por la Norma Técnica Peruana, se envió una muestra para que sea analizada en un laboratorio. Sus resultados se presentan en el cuadro 4 . 
Cuadro 4

Resultado de los análisis físicos y químicos

\begin{tabular}{|c|c|c|c|c|c|}
\hline Requisitos físicos y químicos & Unidad & Mínimo & Máximc & o Resultado & Conclusión \\
\hline Grado alcohólico volumétrico a $20 / 20^{\circ} \mathrm{C}$ & $\%$ & 38 & 48 & 40,5 & $\begin{array}{l}\text { Conforme a NTP } \\
211.0012006\end{array}$ \\
\hline Extracto seco a $100^{\circ} \mathrm{C}$ & $g / l$ & - & 0,6 & 0,04 & $\begin{array}{l}\text { Conforme a NTP } \\
211.0012006\end{array}$ \\
\hline Acetato de etilo & $\mathrm{mg} / 100 \mathrm{ml} \mathrm{AA}$ & 10 & 280 & 56,7 & $\begin{array}{l}\text { Conforme a NTP } \\
211.0012006\end{array}$ \\
\hline Furfural & $\mathrm{mg} / 100 \mathrm{ml} \mathrm{AA}$ & - & 5 & No se detectó & $\begin{array}{l}\text { 5 Conforme a NTP } \\
211.0012006\end{array}$ \\
\hline Aldehidos, como acetaldehido & $\mathrm{mg} / 100 \mathrm{ml} \mathrm{AA}$ & 3 & 60 & 13,6 & $\begin{array}{l}\text { Conforme a NTP } \\
211.0012006\end{array}$ \\
\hline Alcoholes superiores totales & $\mathrm{mg} / 100 \mathrm{ml} \mathrm{AA}$ & 60 & 350 & 323,6 & $\begin{array}{l}\text { Conforme a NTP } \\
211.0012006\end{array}$ \\
\hline Acidez volátil, como ácido acético* & $g / l$ & - & 0,76 & 0,4 & $\begin{array}{l}\text { Conforme a NTP } \\
211.0012002\end{array}$ \\
\hline Alcohol metílico & $\mathrm{mg} / 100 \mathrm{ml} \mathrm{AA}$ & 4 & 100 & 78 & $\begin{array}{l}\text { Conforme a NTP } \\
211.0012006\end{array}$ \\
\hline
\end{tabular}

* La NTP 211.0012006 no establece el límite superior en g/l, por ello se toma como referencia la NTP 211.0012002.

Fuente: Informe de ensayos $N^{\circ}$ 000385-2008 realizado en La Molina Calidad Total, Laboratorio de la Universidad Nacional Agraria La Molina.

Elaboración propia.

\section{INCREMENTO DE LA PRODUCTIVIDAD}

En el cuadro 5 se muestran las cifras de la productividad de la uva en la producción del pisco con maceración y sin maceración.

\section{Cuadro 5}

Cálculo del incremento de la productividad de la uva

\begin{tabular}{ll}
\hline Concepto & \multicolumn{1}{c}{ Datos y resultado } \\
\hline Productividad de la prueba 1 & 0,1128 litros de pisco $/ \mathrm{kg}$ de uva \\
Productividad de la prueba 2 & 0,1703 litros de pisco $/ \mathrm{kg}$ de uva \\
Incremento de productividad & $50,98 \%$ \\
\hline
\end{tabular}

Elaboración propia. 


\section{EVALUACIÓN ECONÓMICA Y CONCLUSIÓN}

La mejora propuesta en el proceso de producción de pisco se concreta en el beneficio económico alcanzado, como se evidencia en los siguientes datos:

Ahorro en cantidad de uva por litro de pisco obtenido:

- Según la prueba 1 se necesitan $8,8679 \mathrm{~kg}$ de uva/litro de pisco.

- Según la prueba 2 se necesitan 5,8726 kg de uva/litro de pisco.

- El ahorro en $\mathrm{kg}$ de uva es 2,9953 kg de uva/litro de pisco.

Si se considera un costo de S/.1,12 por kg de uva, correspondiente a un viñedo en su primer año de producción, se calcula un ahorro en dinero de: 2,9953 x 1,12 = S/. 3,35/litro de pisco. Es importante precisar que el costo por kilogramo de uva baja conforme el viñedo alcanza su máxima producción.

Finalmente, se concluye que el incremento de $50,98 \%$ en la productividad de la uva origina un ahorro de S/.3,35 por cada litro de pisco.

\section{BIBLIOGRAFÍA}

Betalleluz Pallardel, Lincoln. Manual de electricidad básica para ingenieros. Lima: Universidad de Lima, 2003.

Conapisco [en línea]. <http://www.conapisco.org.pe>.

Indecopi. Norma Técnica Peruana NTP 211.001. Bebidas alcohólicas. Pisco. Requisitos. Lima, 2006.

Schuler, Johnny. Pasión por el pisco. Lima: Quebecor World Perú S.A., 2004. 